\title{
Hemodiálisis a un paciente en tratamiento con yodo radioactivo l-131
}

\author{
Ana Vasco Gómez, Martí Lacruz Bassols, Ernestina Junyent Iglesias \\ Servicio de Nefrología y Servicio de Protección Radiológica. Hospital del Mar. Barcelona. España
}

\section{Introducción}

El Yodo 131 es un isotopo radiactivo que se usa con fines médicos, y se ingiere en forma de líquido o cápsula. Su absorción se produce en el tracto gastrointestinal, pasa al torrente sanguíneo y se concentra finalmente en la glándula del tiroides destruyendo sus células ${ }^{1,2}$.

Es un tratamiento de elección en pacientes que estén afectados por un cáncer de tiroides o sufran de un hipertiroidismo $0^{1,2,3}$.

En el cáncer de tiroides se administran dosis muy altas de este radiofármaco (del orden de $100 \mathrm{mCi}$ de actividad $)^{1,2}$, por lo cual el paciente precisa de ingreso hospitalario y medidas de protección radiológicas estrictas, tales como habitación plomada, visitas controladas, etc ${ }^{2,4}$.

En el tratamiento del hipertiroidismo se administran unas dosis bajas de radioyodo (aproximadamente 10 $\mathrm{mCi}$ de actividad) por vía oral en capsulas ${ }^{1,2,3}$.

La máxima captación en la glándula tiroides se produce las primeras 48 horas y la eliminación de la actividad radioactiva remanente es por medio del propio decaimiento físico del isotopo, y a través de orina, heces, sudor y saliva. Es un tratamiento que se administra de forma ambulatoria, aunque el paciente debe seguir una serie de recomendaciones de protección radiológica ya que, debido a la incorporación del radioisótopo, pasa a ser un emisor de radiación ${ }^{1,4}$.

En el hospital del Mar se dan las siguientes recomendaciones que deberá seguir el paciente los primeros 3-4 días post-administración:

\section{Correspondencia: \\ Ana Vasco Gómez}

Calle Gran Vía, 1011, $2^{\circ}-1^{\mathrm{a}}$. 08020 Barcelona

E-mail: avascogo@gmail.com
- No dormir acompañado.

- No estar en contacto con niños ni con mujeres en estado de gestación.

- Utilizar utensilios de comer de un solo uso.

- Beber mucha agua al día y evitar sal yodada.

- En el urinario, tirar de la cadena 3 veces para diluir al máximo la concentración radioactiva.

- Lavar la ropa separada del resto de la familia.

El caso clínico que nos ocupa es el de un paciente con IRC en hemodiálisis al que se le administró yodo 131 para el tratamiento del hipertiroidismo.

La legislación actual distingue entre los profesionales expuestos (personal sometido a exposición radiológica debido a su trabajo diario) y los no expuestos o miembros del público (personas que pueden estar expuestas a una radiación no generada en su trabajo diario). El límite anual de dosis de radiación para unos y otros es diferente, está regulado por el Reglamento de Protección Sanitaria contra radiación ionizante ${ }^{5}$. (Tablal).

Tabla 1. Límite anual de dosis efectiva para el personal no expuesto y el expuesto.

\section{Límite anual de dosis efectiva en cuerpo entero \\ Profesionales NO expuestos (=miembros del público) \\ Profesionales expuestos \\ $1 \mathrm{mSv} / \mathrm{año} 0^{(1)}$ \\ $20 \mathrm{mSv} / \mathrm{año}$}

(1) MiliSievert/año.

La dosis estimada según el cálculo teórico descrito a continuación que puede recibir el /la profesional sanitaria que esté al cuidado del paciente así como el resto de pacientes presentes durante la sesión de hemodiálisis podría llegar a ser de 0,1-0,2 mSv. Este valor, representa un 10-20\% del Límite Anual de Dosis, por tratarse en ambos casos de personal profesional NO expuesto/ miembros del público.

A pesar de tratarse de un valor alejado del límite vigente, en base al criterio ALARA (As Low As Reaso- 
nably Achievable $)^{6,7}$ aplicable a la exposición radiológica, el riesgo radiológico será siempre "Tan bajo como sea razonablemente posible".

El objetivo de presentar este caso clínico es el describir la realización del tratamiento de HD en un paciente emisor de radiación por tratamiento con Yodo 131 y que nos llevó a elaborar un protocolo de actuación conjuntamente con el Servicio de Protección Radiológica de nuestro hospital para disminuir la exposición a la radiación que genera este paciente durante las sesiones de Hemodiálisis de la primera semana posttratamiento con yodol31, tanto en el personal sanitario como en el resto de pacientes de la sala.

\section{Caso clínico}

El paciente varón de 38 años, natural de Nigeria. Antecedentes patológicos:

- HTA.

- Infección por VIH.

- Varias infecciones oportunistas.

- ERCT. Inició HD el 28/6/2007. Su turno habitual es el de Martes-Jueves-Sábado.

- Hipertiroidismo severo y de difícil control.

El paciente percibió $10 \mathrm{mCi}$ de ${ }_{53}^{131} \mathrm{I}$ (Yodo). Para poder determinar el riesgo al que estaría sometido tanto el personal sanitario, así como el resto de pacientes en tratamiento se realizó un cálculo teórico previo estimativo de la dosis integrada efectiva (Hin) que produce el paciente durante el tiempo $\left(t+t^{\prime}\right)$ del tratamiento de $H D$, una vez administrada la dosis de radiofármaco. (Figural).

$$
H_{t n}=\int_{t}^{t+t^{\prime}}(H) d t=\int_{t}^{t+t^{\prime}}\left(H_{0} e^{-\lambda t}\right) d t=H_{0} \int_{t}^{t+t^{\prime}}\left(e^{-\lambda t}\right) d t=\frac{-H_{0}}{\lambda}\left[e^{-\lambda t}\right]^{t+t^{\prime}}=\frac{H_{0} T_{1 / 2}}{\ln 2}\left[1-e^{-\lambda t}\right]
$$

Figura 1. Fórmula para el cálculo teórico de la dosis integrada efectiva que produce el paciente durante el tratamiento de HD.

Se programó las Hemodiálisis (HD) de manera que el paciente ingirió la dosis de Yodo post HD el jueves a las $13 \mathrm{~h}$ y la siguiente HD se realizo el domingo a partir de las 15h, retomando su turno normal de Martes-jueves-sábado la semana siguiente. Se considero a las diálisis del domingo y martes, como de riesgo por radiación.

A fin de minimizar la exposición a la que se podían ver afectados los presentes en la sala, se activaron medidas de protección sobre tres variables:
- Tiempo de exposición.

- Distancia a la fuente de radiación.

- Blindaje.

\section{Tiempo y distancia}

La proximidad con la fuente emisora, en este caso el paciente, puede tener una duración variable que sin incidencias podemos establecer en 20 minutos durante la conexión y desconexión al monitor de hemodiálisis. Para disminuir el riesgo del personal se decidió que la enfermera que conectara al paciente fuera diferente a la que lo desconectara, así como, durante el resto de la sesión, mantener una distancia de seguridad mínima de 2 metros al paciente siempre que fuera posible.

\section{Blindaje}

Como hemos señalado, aun diseñando estrategias para intentar controlar el tiempo y la distancia a la fuente emisora, al tratarse de un paciente al que se le está realizando un tratamiento, pueden darse situaciones que requieran actuaciones de emergencia que den lugar a una proximidad, o incluso contacto, con el foco emisor. Por este motivo se optó por la utilización de una serie de materiales para minimizar la dosis de radiación al que estaba expuesto el personal.

Se dispuso de una mampara de espesor equivalente 0,5 $\mathrm{mm}$ de $\mathrm{Pb}$ para la separación del paciente con el personal.

Se colocó al paciente un protector plomado de tiroides que al ser el órgano de máxima captación del radioisótopo actuaría como blindaje sobre el lugar de mayor emisión de la radiación.

Se habilitó un chaleco plomado para utilizarlo el personal durante conexión y desconexión (período de máxima proximidad) y en caso de una situación de emergencia que requiera acciones sobre el paciente.

Somos conscientes de que los materiales plomados utilizados no están preparados para optimizar la atenuación en este caso debido a que la energía gamma del Yodo 131 es superior a la energía estándar para la cual se diseñan dichos blindajes. No obstante, en aplicación una vez más del criterio A.L.A.R.A de mínima dosis razonablemente factible, decidimos el uso de dichas protecciones.

\section{Resultados}

Al llegar el paciente a la sala de hemodiálisis se llevaron a cabo varias mediciones. 
En primer lugar se procedió a medir la radiación proveniente del paciente sin ningún tipo de protección. Una vez comprobado el nivel basal de radiación se coloco el protector plomado en la tiroides, y volvió a repetir la medición.

Al conectar el paciente, se tomaron medidas del circuito extracorpóreo y sobretodo del dializador, que a priori era donde se podía encontrar mayor nivel de radiación después del propio paciente.

Por último una vez finalizada la sesión de hemodiálisis se realizaron de nuevo las mediciones para comprobar la existencia, o no, de residuo radioactivo. (Tablas 2 y 3).

Las mediciones se llevaron a cabo con el detector:

Lamse MS60 dotado con una sonda específica (RDIL) para medida de la tasa de dosis y con una sonda específica (CT15) para la medida de contaminación.

Tabla 2. Tasa de dosis obtenida en el control de enfermería con el paciente sin protección y con protección.

\begin{tabular}{l|l|l}
\hline $\begin{array}{l}\text { Tasa de dosis medida } \\
\text { en control de enfermería }\end{array}$ & Sin protección & Con protección \\
\hline
\end{tabular}

(1) MicroSievert/hora.

Tabla 3. Medida de la contaminación del material.

\begin{tabular}{l|l|l}
\hline & Pre-HD & Post-HD \\
\hline Dializador & Fondo $^{(1)}$ & NA $^{(2)}$ \\
\hline Circuito Extracorpóreo & Fondo & NA \\
\hline
\end{tabular}

(1) Se entiende por "Fondo" el valor de contaminación debido al fondo radiactivo natural ( $<2$ cuentas por segundo).

(2) NA: El valor medido no sufrió alteraciones apreciables.

\section{Conclusiones}

Una vez analizadas las medidas de radiación generadas por el paciente y en los materiales utilizados para la hemodiálisis podemos concluir:

- La contaminación del material es prácticamente despreciable y, de producirse, se limitaría al dializador.

- Colocar al paciente protector plomado de tiroides fue muy eficaz, ya que es en la glándula donde se concentro la mayor cantidad de radiación, fue la medida que más redujo la emisión.

- Alargar el tiempo entre la toma de la cápsula radioactiva y la Hemodiálisis el máximo posible para respetar las 72 horas de decaimiento radiológico.
- Mantener una distancia de seguridad con la fuente emisora mínima de 2 metros permite dializar a otro paciente en la misma sala sin ningún riesgo.

- Habilitar chalecos de plomo para las posibles actuaciones de emergencia sobre el paciente que requieran proximidad prolongada.

- Rotación del personal de enfermería. Esta medida minimiza la exposición individual durante la conexión y desconexión y en caso de actuaciones de emergencia.

- Como medida de protección para el personal se comprobó que no sería necesario utilizar la mampara. El blindaje de tiroidal dispuesto en el emisor y la distancia de seguridad establecida, son mecanismos suficientes para garantizar la seguridad radiológica de los profesionales sanitarios y el resto de pacientes de la sala.

- Destacar finalmente que, aun no siendo habitual la hemodiálisis de un paciente contaminado radiológicamente, es una situación que puede repetirse en más de una ocasión. Por este motivo, minimizar la dosis absorbida del personal de enfermería que realiza el tratamiento puede ser útil como medida preventiva ante una posible reincidencia del suceso.

\section{Recibido: 20 enero 2016 \\ Revisado: 4 febrero 2016 \\ Modificado: 27 febrero 2016 \\ Aceptado: 3 marzo 2016}

\section{Bibliografía}

1. Consejo de Seguridad Nuclear, Sociedad Española de Protección Radiológica y Sociedad Española de Física Médica. Documento del Foro sobre protección radiológica en el medio sanitario: Criterios de alta de pacientes y medidas para la protección radiológica del público después de tratamientos metabólicos con I131. 2011 0ct [citado 2015 0ct 24]. Disponible en: http://www.sepr.es/html/recursos/descargables/I-131-PR-alta-pacientes.pdf

2. González Rivero Levi, Turcios Tristá Silvia Elena, Velasco Mirabal Milagros. Aplicaciones clínicas del radioyodo 131 (I131) en las enfermedades del tiroides. Rev Cubana Endocrinol 
[revista en la Internet]. 2012 Dic [citado 2015 Oct 24] ; 23(3): 256-263. Disponible en:http:// scielo.sld.cu/scielo.php?script=sci_arttext\&pi$d=$ S1561-29532012000300011\&lng=es

3. Gallegos-Villalobos Ángel, García-López Fernando, Escalada Carmen, Ortiz Juan J., Cardona Jorge, Medina Amparo et al . Uso de yodo radiactivo I-131 y monitorización de radiactividad en pacientes con enfermedad renal crónica en hemodiálisis. Nefrología (Madr.) [revista en la Internet]. 2014 [citado 2015 0ct 24]; 34(3): 317-322. Disponible en: http://scielo.isciii.es/scielo.php?script=sci_art text\&pid=S0211-69952014000300006\&lng=es. http://dx.doi.org/10.3265/Nefrologia.pre2014. Jan. 12442

4. IAEA Release of Patients After Radionuclide Therapy. Safety Report Series No.63. 2009 0ct. [citado 2015 0ct 24]. Disponible en: http://www-pub. iaea.org/MTCD/Publications/PDF/pub1417_web. pdf
5. Real Decreto 783/2001 de 6 de Julio, por el que se aprueba el Reglamento sobre protección sanitaria contra radiaciones ionizantes. BOE núm. 178 de 26 de julio de 2001. [citado 2015 0ct 24]. Disponible en: http://www.boe.es/diario_boe/txt.php?i$d=B 0 E-A-2001-14555$

6. ICRP, 1977. Recommendations of the ICRP. ICRP Publication 26. Ann. ICRP 1 (3).

7. 2007 Recommendations of the International Commission on Radiological Protection. ICRP.Publication 103. Ann. ICRP 37 (2-4), 2007. 